\title{
Efecto de las fases lunares en la siembra, trasplante y producción de la lechuga (Lactuca sativa) orgánica en la E.E.A. El Mantaro de la UNCP
}

\author{
Effect of the lunar phases in the sowing, transplanting and production \\ of organic lettuce (Lactuca sativa) in the A.E.S. El Mantaro of the UNCP
}

\author{
aMata Adauto, Z.; Bonilla Mancilla, H.; Concha Salazar, J. \\ Facultad de Agronomía / Universidad Nacional del Centro del Perú \\ Email: zmata@uncp.edu.pe
}

\section{Resumen}

La producción de lechuga y su rendimiento dependen de factores agronómicos, ambientales y otros; en ese sentido, uno de los factores, tal vez no tomados en cuenta por el productor actual, es el posible efecto de las fases lunares. El objetivo fue determinar el efecto de las fases lunares en la siembra, trasplante y producción de la lechuga (Lactuca sativa) en la Estación Experimental Agropecuaria El Mantaro de la UNCP. Para este fin, se almacigaron semillas de dos cultivares (Iceberg y Rizada) y trasplantaron dichas plántulas en terreno definitivo durante las cuatro fases lunares: Luna Nueva, cuarto creciente, Luna Llena y cuarto menguante en los momentos partiles; es decir, al inicio de cada fase lunar. Se empleó el diseño de block completamente randomizado llevado al modelo factorial $2 \times 4$ con 3 repeticiones.

Los cultivares fueron abonados orgánicamente, tanto en la preparación de suelo como en los almacigamientos, y trasplantes a terreno definitivo. Los resultados, indican que las siembras, trasplantes y producción obtenidas en la fase de Luna Llena ocuparon el primer puesto, tanto en altura de planta como en producción con un promedio de $4708 \mathrm{~kg} / \mathrm{ha}$; en segundo lugar, se ubica la fase de cuarto creciente en altura de planta y producción con un promedio de $3458 \mathrm{~kg} / \mathrm{ha}$; en tercer lugar, se ubica la Luna Nueva en altura de planta y producción con un promedio de $2945 \mathrm{~kg} / \mathrm{ha}$ y; en último lugar, la fase de cuarto menguante en altitud de planta y producción media de $2387 \mathrm{~kg} / \mathrm{ha}$.

Palabras clave: fases lunares, cosmobiología, producción lechuga, desarrollo vegetativo

\begin{abstract}
The production of lettuce and its yield depend on agronomic, environmental and other factors, in this sense one of the factors perhaps not taken into account by the current producer is the possible effect of the lunar phases. In this respect, our objective was to determine the effect of the lunar phases in the sowing, transplantation and production of lettuce (Lactuca sativa) in the El Mantaro agricultural experimental station of the UNCP. For which seeds of two cultivars (Iceberg and Curly) were stored and transplanted these seedlings in definitive terrain during the four lunar phases: New Moon, Crescent Moon, Full Moon and Quarter Waning in the partiles moments; that is, at the beginning of each lunar phase. The completely randomized block design, carried to the $2 \times 4$ factorial model with 3 repetitions was used.

The cultivars were organically fertilized both in the preparation of soil and in storage and transplants to permanent ground. The results indicate that the plantings, transplants and production obtained in the full moon phase occupied the first place both in plant height and in production with an average of $4708 \mathrm{~kg} / \mathrm{ha}$, in second place is the Quarter Crescent phase in height of plant and production with an average of $3458 \mathrm{~kg} /$ ha, in third place New Moon in height of plant and production with an average of $2945 \mathrm{~kg} /$ ha and in fourth and last place the phase of waning in altitude of plant and average production of $2387 \mathrm{~kg} / \mathrm{ha}$.
\end{abstract}

Keywords: lunar phases, cosmobiology, lettuce production, vegetative development 


\section{Introducción}

En la producción y rendimiento de la lechuga (Lactuca sativa L.), que es una hortaliza muy importante en la alimentación humana, intervienen muchos factores para un buen rendimiento; y uno de esos factores, tal vez no tomados en cuenta por el productor actual, es la influencia de las fases lunares, que nuestros ancestros y las ciencias ancestrales, como la cosmobiología, recomiendan tomar en cuenta dichos cambios lunares, con el propósito de incrementar la producción, no sólo de la lechuga como es el caso, sino aplicable a toda los cultivos de la producción agraria; tal como lo expresa Raynaud (1975), quien menciona que: "En lo concerniente a las simientes y a los cultivos, buen número de cultivadores han obtenido los mejores resultados siguiendo las reglas astrológicas. Preparar la tierra, cultivar y humear, mientras que la luna se encuentra en Carnero, Gemelos, León, Virgen, Sagitario o Acuario. Sembrar en particular los guisantes, frijoles, coles durante la Luna nueva de marzo y hasta la Luna llena...Las zanahorias, cebollas, rábanos y todas las raíces, pueden ser sembrados desde la Luna llena hasta la Luna nueva. El fresal, ranunculáceas, etc... durante la Luna creciente".

Desde tiempos antiguos, los agricultores observaron que las fases de la Luna influyen en la producción de los cultivos, estimulando la rápida germinación o retrasándola, sobretodo en la agricultura ecológica donde las plantas crecen de forma natural, sin el uso de estimulantes químicos. La influencia de las fases de la Luna en la productividad y en la calidad de los cultivos, se manifiesta a través del ascenso o descenso de la savia (alimento de la planta); al parecer, la luz proveniente de la luna, según la intensidad propia de cada fase, interviene en la germinación y desarrollo vegetativo, esto por la influencia de la irradiación lunar. ECO agricultor (2011).

Palomino y Mata (1980) reportan que las fases lunares si influyen en el incremento del rendimiento del tubérculo de papa, obteniéndose los siguientes resultados: Primer lugar, cuarto menguante con 18,11 tm/ha; segundo lugar, cuarto creciente con 17,36 tm/ha; tercer lugar, con 12,19 tm/ha y; en cuarto lugar, Luna llena con $10,97 \mathrm{tm} / \mathrm{ha}$.

La hortaliza en estudio, es de periodo vegetativo anual, corresponde a la familia de las compuestas, inflorescencia en cabezuela, procede del entorno del Mediterráneo en Asia Menor, es una hortaliza de consumo masivo (Tarigo et al., 2004).

Considerando que las plantas tienen savia, estas sufren la atracción de la Luna, incluyendo el Sol y la superficie de la Tierra (se da en periodos de sus influencias). Esto se debe a la fuerza gravitacional que ejerce la Luna con respecto a la Tierra, sobre todo en el elemento agua. (Restrepo, 2005 citado por Torres, 2012):
- Cuarto creciente y Luna llena: Sembrar, aporcar y cosechar tubérculos y raíces. Preparación de tierra.

- Luna llena: Sembrar, aporcar y cosechar hojas y granos frescos. Recolectar flores y frutos.

- Luna nueva: Preparar tierra, sembrar y cosechar granos secos y abonar las plantas (Calendario Lunar Cosmobiológico, 2011).

\section{Cultivo de la lechuga}

La Lechuga (Lactuca sativa L.) es una planta anual que pertenece a la familia compositae. El centro de origen primario de esta especie se ubica en Asia Menor, en las proximidades del Mar Mediterráneo. Es una de las hortalizas más importantes de las que se consumen crudas (Tarigo et al., 2004). En el Perú también reviste esta importancia, siendo cultivada todo el año, concentrándose la producción en el valle del Mantaro y, en menor magnitud, en los alrededores de otros centros poblados.

En tal sentido, el objetivo fue determinar el efecto de las fases lunares en la siembra, trasplante y producción de la lechuga.

\section{Caracterización de la especie}

El sistema radicular es denso y superficial. Normalmente es pivotante, alcanzando una profundidad máxima de $60 \mathrm{~cm}$, con numerosas raíces laterales en los primeros $30 \mathrm{~cm}$. Si el cultivo se lleva a cabo mediante la modalidad de almácigo y trasplante, se rompe la dominancia apical y hay fácil regeneración de raíces adventicias, resultando un sistema radicular más ramificado y superficial. (Galván y Rodríguez, 1999 citados por Tarigo et al., 2004).

Esta hortaliza es de suma importancia en la alimentación humana; en nuestro medio, se destina una parte de su cultivo al consumo fresco. La demanda cada vez mayor de producción de alimentos por parte de la población siempre creciente de consumidores y decreciente de productores, destaca la importancia de aumentar la productividad de los cultivos, mejorando las condiciones físicas y nutritivas del suelo, ya que es de donde extrae los nutrientes que necesita para su crecimiento; así como, considerar los factores ambientales (FCA-UNA et al., 2005 citado por López, 2013).

\section{Requerimientos del cultivo}

Germinación: Temperatura óptima entre 15 y $20{ }^{\circ} \mathrm{C}$, humedad relativa: de 60 a $80 \%$ (Tarigo et al., 2004).

Desarrollo vegetativo: Temperaturas de 14 a $18{ }^{\circ} \mathrm{C}$ durante el dia y de 5 a $8{ }^{\circ} \mathrm{C}$ por la noche. La lechuga se adapta mejor a climas frescos y húmedos (Tarigo et al., 2004).

\section{Fases de crecimiento de la lechuga}

Fase de plántula: Comprende desde la emergencia a la aparición de la tercera o cuarta hoja verdadera. Esta etapa dura de 3 a 6 semanas en función de las condiciones ambientales (especialmente temperatura). 
Fase de roseta: Empieza a disminuir la relación largo/ancho de las láminas foliares. Los pecíolos se hacen sumamente cortos o desaparecen, por lo que la planta adquiere aspecto de roseta. En esta etapa la planta llega a 12 - 14 hojas verdaderas, (Tarigo et al., 2004).

\section{Las fases lunares}

Las fases lunares que se inician con la Luna nueva, cuarto creciente, Luna llena y cuarto menguante, se relacionan a la fuerza gravitacional del satélite con respecto a la Tierra; estas se traducen en que la savia de las plantas asciende hacia la parte media y superior de las plantas, siendo mayor en la Luna llena; entre tanto, en la fase cuarto menguante, la sabia de la parte superior y media bajan al tallo y al sistema radicular de las plantas. Bajo este contexto, la fisiología de las plantas se desarrolla más o estas bajan, según los flujos lunares (Torres, 2012)

Según científicos investigadores, afirman que el efecto de las mareas se expresan en forma muy notable en el crecimiento rápido de las plantas, el cual es conocido como el flujo y experimenta un retraso en su desarrollo las plantas cuando atraviesan un reflujo que correspondería al cuarto menguante (Torres, 2012).

\section{Métodos y materiales}

\section{Material genético en estudio}

El material genético en estudio estuvo conformado por 2 cultivares de lechuga (Lactuca sativa $\mathrm{L}$ ): C1 = Iceberg romana (cabezuela) y C2 $=$ Rizada (Seda).

\section{Fases lunares en estudio}

Se identificaron como: F1 = Luna nueva F2 = Cuarto creciente F3 = Luna llena F4 = Cuarto menguante.

Las cobinaciones correspondientes a las variedades y fases lunares se muestran en la Tabla 1.

Tabla 1

Tratamientos en estudio

\begin{tabular}{ccc}
\hline Tratamiento & Código & Combinación \\
\hline 1 & F1C1 & Luna Nueva - Iceberg \\
2 & F1C2 & Luna Nueva - Rizada \\
3 & F2C1 & Cuarto Creciente - Iceberg \\
4 & F2C2 & Cuarto Creciente - Rizada \\
5 & F3C1 & Luna Llena - Iceberg \\
6 & F3C2 & Luna Llena - Rizada \\
7 & F4C1 & Cuarto Menguante - Iceberg \\
8 & F4C2 & Cuarto Menguante - Rizada \\
\hline
\end{tabular}

\section{Diseño metodológico}

El estudio consistió en la siembra y trasplante de los 2 cultivares de lechuga en cada fase lunar (4 fases: Luna nueva, cuarto creciente, Luna llena y cuarto menguante), y como materia orgánica se empleó el estiércol de vacuno preparado. La siembra se efectuó en camas almacigueras. El trasplante se efectuó en terreno definitivo.

\section{Siembras en camas almacigueras, según fases lunares}

Cuarto creciente: 26/12/2017; Luna llena: 01/01/2018; Cuarto menguante: 08/01/2018 y Luna nueva: $16 / 01 / 2018$.

\section{Trasplante de plántulas de lechuga, según fases lunares}

Cuarto creciente: 23/02/2018; Luna llena: 01/03/2018; Cuarto menguante: 09/03/2018 y Luna nueva: 17/03/2018.

\section{Diseño experimental}

La distribución de los tratamientos en estudio estuvo dispuesta en un diseño estadístico de Bloques Completamente Randomizados (BCR) con arreglo factorial.

$$
\mathrm{Xijk}=\mu+\delta \mathrm{k}+\tau \mathrm{i}+\beta \mathrm{j}+(\tau \beta) \mathrm{ij}+\varepsilon \mathrm{ijk}
$$

Dónde: Xijk = Observación cualquiera dentro del experimento; $\mu=$ Media poblacional; $\delta \mathrm{k}=$ Efecto aleatorio del k-ésimo bloque o repetición; $\tau \mathrm{i}=$ Efecto aleatorio del i-ésimo nivel del factor $\mathrm{A} ; \beta \mathrm{j}=$ Efecto aleatorio del j-ésimo nivel del factor $\mathrm{B} ;(\tau \beta) \mathrm{ij}=$ Efecto aleatorio del i-ésimo nivel del factor $A$ interaccionando con j-ésimo nivel del factor B y Eijk = Error experimental

Asimismo, i toma valores de $1,2,3, \ldots$ hasta a niveles del factor A; $j$ toma valores de 1, 2, 3,... b niveles de factor $\mathrm{B}$; $\mathrm{k}$ toma valores de $1,2,3, \ldots \mathrm{r}$ bloques o repeticiones.

\section{Resultados y discusión}

\section{Altura de planta}

En la Tabla 2, del análisis de varianza de la altura de planta, se observa que, en la fuente de bloques no existe diferencia estadística significativa, debido a que no hay efecto ambiental que altera esta variable dentro del experimento.

En la fuente del Factor A (fases lunares), existe diferencia estadística altamente significativa, debido a que hay influencia de los niveles de este factor que dan respuestas diferentes en esta variable.

En la fuente del Factor B (cultivares), no muestran significación estadística, debido a que los niveles dieron respuestas similares para esta característica y, en la fuente de la interacción $\mathrm{AxB}$ (fases lunares $\mathrm{x}$ cultivares) no muestran significación estadística, debido a que los niveles de cada factor actuaron en forma independiente.

El coeficiente de variabilidad de 8,50\% es considerado como "muy bajo" (Osorio, 2000), el que indica que, dentro de cada tratamiento la altura de planta fue muy homogénea. 
Tabla 2

Análisis de varianza de la altura de planta del efecto de las fases lunares en la producción ecológica de dos cultivares de lechuga. Dónde $S=0,476$, promedio es 0,506 y C.V. $=8,50 \%$

\begin{tabular}{cccccccc}
\hline Factores & $\begin{array}{c}\text { Grados de } \\
\text { libertad }\end{array}$ & S.C. & C.M. & Fc. & F0,05 & F0,01 & Signif. \\
\hline Bloques & 2 & 0,108 & 0,054 & 0,238 & 3,739 & 6,515 & n.s. \\
A (fases lunares) & 3 & 26,212 & 8,737 & 38,538 & 3,344 & 5,564 & $* *$ \\
B (cultivares) & 1 & 0,960 & 0,960 & 4,234 & 4,600 & 8,862 & n.s. \\
AXB & 3 & 0,276 & 0,092 & 0,406 & 3,344 & 5,564 & n.s. \\
Error & 14 & 3,174 & 0,227 & & & & \\
Total & 23 & 30,731 & & & & & \\
\hline
\end{tabular}

En la Tabla 3, de la prueba de significación de los promedios de la altura de planta para los niveles del Factor A (fases lunares), se observa que el nivel a3 (Luna llena) ocupa el primer lugar con un promedio de 6,777 $\mathrm{cm}$ de altura y supera estadísticamente a los demás niveles; esto debido a que en esta fase la savia de las plantas asciende hacia la parte media y superior de las plantas, siendo mayor en la Luna llena; favoreciendo el crecimiento de las plantas de lechuga; entre tanto, en la fase cuarto menguante, la savia de la parte superior y media bajan al tallo y al sistema radicular. Los promedios de la altura de planta oscilaron desde 3,915 hasta $6,777 \mathrm{~cm}$ respectivamente. Para los niveles del Factor B (cultivares), se observa que los niveles b2 (Seda) y b1 (Iceberg) presentan promedios de 5,805 y 5,405 $\mathrm{cm}$ respectivamente, y no muestran significación estadística entre ellos, debido a que estos cultivares tienen caracteres similares en su crecimiento y desarrollo.

Tabla 3

Prueba de significación de Tukey de los promedios de la altura de planta para los niveles del Factor $A$ (Fases lunares), $A L S(T) 0,05=0,799, y$ Factor B (cultivares), $A L S(T) 0,05=0,416$

\begin{tabular}{ccccc}
\hline Factor & O.M. & Nivel & Promedio & Significación \\
\hline & 1 & $\begin{array}{c}\text { a3 } \\
\text { (Luna llena) }\end{array}$ & 6,777 & a \\
A & 2 & $\begin{array}{c}\text { a } 2 \\
\text { (cuarto creciente) }\end{array}$ & 5,917 & b \\
& 3 & $\begin{array}{c}\text { a1 } \\
(\text { Luna nueva) }\end{array}$ & 5,812 & b \\
& 4 & $\begin{array}{c}\text { a } 4 \\
\text { (cuarto menguante) }\end{array}$ & 3,915 & c \\
B & 1 & $\begin{array}{c}\text { b2 } \\
\text { (seda) }\end{array}$ & 5,805 & a \\
& 2 & $\begin{array}{c}\text { b1 } \\
\text { (iceberg) }\end{array}$ & 5,405 & a \\
\hline
\end{tabular}

En la Tabla 4, de la prueba de significación de los promedios de la altura de planta para las interacciones AxB (fases lunares x cultivares), se observa que las 6 primeras interacciones, según el orden de mérito, no muestran significación estadística entre ellos por presentar res- puestas similares en esta variable; sin embargo, muestran significación estadística con las dos últimas interacciones, debido a que en esta fase (cuarto menguante) hay menos ascenso de agua a la planta y menor luminosidad nocturna, no favoreciendo el crecimiento y desarrollo de la planta.

\section{Tabla 4}

Prueba de significación de Tukey de los promedios de la altura de planta, para la interacción $A \times B$

(Fases lunares $x$ cultivares), $A L S(T) 0,05=1,372$

\begin{tabular}{cccc}
\hline OM & Interacción & Promedio & Significación \\
\hline 1 & $\mathrm{a} 3 \mathrm{~b} 2$ & 6,847 & $\mathrm{a}$ \\
2 & $\mathrm{a} 3 \mathrm{~b} 1$ & 6,707 & $\mathrm{a}$ \\
3 & $\mathrm{a} 2 \mathrm{~b} 2$ & 6,217 & $\mathrm{a}$ \\
4 & $\mathrm{a} 1 \mathrm{~b} 2$ & 6,123 & $\mathrm{a}$ \\
5 & $\mathrm{a} 2 \mathrm{~b} 1$ & 5,617 & $\mathrm{a}$ \\
6 & $\mathrm{a} 1 \mathrm{~b} 1$ & 5,500 & $\mathrm{a}$ \\
7 & $\mathrm{a} 4 \mathrm{~b} 2$ & 4,033 & $\mathrm{~b}$ \\
8 & $\mathrm{a} 4 \mathrm{~b} 1$ & 3,797 & $\mathrm{~b}$ \\
\hline
\end{tabular}

En la Tabla 5, del análisis de varianza del peso de cabeza de lechuga; se observa que en la fuente de bloques, no existe diferencia estadística significativa, debido a que no hay efecto ambiental que altera esta variable dentro del experimento. En la fuente del Factor A (fases lunares) existe diferencia estadística significativa, debido a que hay influencia de los niveles de este factor (fases lunares) que dan respuestas diferentes en esta variable. En la fuente del Factor B (cultivares) no muestran significación estadística, debido a que los niveles dieron respuestas similares para esta característica y, en la fuente de la interacción $\mathrm{AxB}$ (fases lunares x cultivares) no muestran significación estadística, debido a que los niveles de cada factor actuaron en forma independiente.

El coeficiente de variabilidad de $20.00 \%$ es considerado como "bajo" (Osorio, 2000), el que indica que dentro de cada tratamiento, los pesos de las cabezas de lechuga fueron homogéneas. 
Tabla 5

Análisis de varianza del peso de cabeza de lechuga del efecto de las fases lunares en la producción ecológica de dos cultivares de lechuga Dónde $S=0,062$, promedio es 0,098 y C.V. $=20,00 \%$

\begin{tabular}{ccccccccc}
\hline Factores & $\begin{array}{c}\text { Grados de } \\
\text { libertad }\end{array}$ & S.C. & C.M. & Fc. & F0,05 & F0,01 & Signif. \\
\hline Bloques & 2 & 0,003 & 0,001 & 0,374 & 3,739 & 6,515 & n.s. \\
A (fases lunares) & 3 & 20,382 & 5,800 & 3,500 & 3,344 & 5,564 & $*$ \\
B (cultivares) & 1 & 0,001 & 0,001 & 0,297 & 4,600 & 8,862 & n.s. & n.s. \\
AXB & 3 & 0,004 & 0,001 & 0,349 & 3,344 & 5,564 & & \\
Error & 14 & 0,054 & 0,004 & & & & & \\
Total & 23 & 0,073 & & & & & \\
\hline
\end{tabular}

En la Tabla 6, de la prueba de significación de los promedios de peso de cabezas de lechuga para los niveles del Factor A (fases lunares), se observa que el nivel a3 (Luna llena) ocupa el primer lugar con un promedio de $1,130 \mathrm{~kg}$ de peso y supera estadísticamente a los demás niveles, debido a que en esta fase, la savia de las plantas asciende hacia la parte media y superior de las plantas, siendo mayor en la Luna llena, favoreciendo a una mayor formación en tamaño de las cabezas de lechuga; entre tanto, en la fase cuarto menguante, la savia de la parte superior y media bajan al tallo y al sistema radicular disminuyendo su desarrollo de cabezas de lechuga. Los promedios de peso oscilaron desde 0,573 hasta $1,130 \mathrm{~kg}$ respectivamente.

En la misma Tabla 6, se muestran los promedios de peso de cabeza de lechuga para los niveles del Factor B (cultivares), se observa que, los niveles b2 (Seda) y b1 (Iceberg) presentan promedios de 0.105 y $0.091 \mathrm{~kg}$ respectivamente, y no muestran significación estadística entre ellos, debido a que estos cultivares tienen caracteres similares en el peso de cabeza de lechuga.

Tabla 6

Prueba de significación de Tukey de los promedios de peso de cabeza de lechuga para los niveles del Factor $A$ (Fases lunares), $A L S(T) 0,05=0,104, y$ Factor B (cultivares), $A L S(T) 0,05=0,054$

\begin{tabular}{ccccc}
\hline Factor & O.M. & Nivel & Promedio & Significación \\
\hline & 1 & $\begin{array}{c}\text { a3 } \\
\text { (luna llena) }\end{array}$ & 1,130 & a \\
A & 2 & $\begin{array}{c}\text { a } 2 \\
\text { (cuarto creciente) }\end{array}$ & 0,830 & b \\
& 3 & $\begin{array}{c}\text { a1 } \\
\text { (luna nueva) }\end{array}$ & 0,707 & b \\
\hline & 4 & $\begin{array}{c}\text { a } 4 \\
\text { (cuarto menguante) }\end{array}$ & 0,573 & c \\
B & 1 & $\begin{array}{c}\text { b2 } \\
\text { (seda) } \\
\text { b1 }\end{array}$ & 0,105 & a \\
\hline
\end{tabular}

En la Tabla 7, de la prueba de significación de los promedios de peso de cabeza de lechuga, para las interacciones AxB (fases lunares x cultivares), se observa que las 6 primeras interacciones, según el orden de mérito, no muestran significación estadística entre ellos, por presentar respuestas similares en esta variable.

Tabla 7

Prueba de significación de Tukey de los promedios de peso de cabeza de lechuga para la interacción $A \times B$ (Fases lunares $x$ cultivares), $A L S(T) 0,05=0,178$

\begin{tabular}{cccc}
\hline OM & Interacción & Promedio & Significación \\
\hline 1 & A2b1 & 0,159 & $\mathrm{a}$ \\
2 & A1b2 & 0,112 & $\mathrm{a}$ \\
3 & A1b1 & 0,102 & $\mathrm{a}$ \\
4 & A2b2 & 0,101 & $\mathrm{a}$ \\
5 & A3b1 & 0,082 & $\mathrm{a}$ \\
6 & A3b2 & 0,081 & $\mathrm{a}$ \\
7 & A4b1 & 0,040 & $\mathrm{~b}$ \\
8 & A4b2 & 0,030 & $\mathrm{~b}$ \\
\hline
\end{tabular}

\section{Conclusiones}

- Las fases lunares tienen efecto en el almacigamiento, trasplante y producción de la lechuga (Lactuca sativa), evidenciado por los resultados obtenidos: así. para el crecimiento en altura de planta en orden de mérito, se reporta en primer lugar: Luna llena, con un promedio de $6.77 \mathrm{~cm}$; en segundo lugar, cuarto creciente con $5.91 \mathrm{~cm}$; en tercer lugar, Luna nueva con $5.81 \mathrm{~cm}$ y; en último lugar, la fase de cuarto menguante con $3.91 \mathrm{~cm}$ de altura.

- Así mismo, en la producción de cabezas de lechuga se evidenció la influencia de las fases lunares, al obtener similares resultados; así, en orden de mérito se reporta; en primer lugar, la 
fase de Luna llena con un promedio de $4708 \mathrm{~kg}$; en segundo lugar, cuarto creciente con $3458 \mathrm{~kg}$; en tercer lugar, Luna nueva con $2945 \mathrm{~kg}$ y; en cuarto lugar, cuarto menguante con $2387 \mathrm{~kg} /$ parcela.

- La fase de Luna llena superó significativamente a las demás, fases tanto en crecimiento en altura de planta como en producción de cabezas de lechuga.

- Las fases de cuarto creciente y Luna nueva, ocuparon el segundo y tercer lugar respectivamente, tanto en crecimiento de altura de planta como en la producción de cabezas de lechuga. Por otra parte, la fase de cuarto menguante ocupó el cuarto y último lugar, tanto en crecimiento de altura de planta como en la producción de cabezas de lechuga, superados significativamente por las demás fases lunares.

\section{Referencias bibliográficas}

Cáceres, (1986). Apuntes de horticultura. 107 p. citado por López Evolio (2013)

López, E. (2013). Fertilización orgánica en el cultivo de la lechuga (Lactuca Sativa L.) Pag.Web (http://abcruraltv.com.ar/producción-la-lechuga -al- tope- delos- Excesos-de-residuos-de-agroquímicos/)

Raynaud De La Ferriére, S. (1975). Propósitos psicológicos. Volumen III, Tomo XVIII, Lima Perú.

Sedano, P. y Mata, Z. (1980). Fenología del cultivo de maíz y las fases lunares.

Tarigo, A; Repeto, C. y Acosta, D. (2004). Evaluación agronómica de biofertilizantes en la producción de lechuga (Lactuca sativa L.) a campo. Tesis, Universidad de la República, Facultad de Agronomía, Uruguay.

Torres, A. (2012). Influencia de la Luna en la agricultura. Tesis, Universidad de Cuenca, Facultad de Ciencias Agrarias, Ecuador. 